\title{
Chromosomal localization and regulation of the longevity determinant genes in a selected strain of Drosophila melanogaster*
}

\author{
STEVEN BUCK†, ROBERT A. WELLS†, STEVEN P. DUDAS†, GEORGE T. BAKER IIIङ \& \\ ROBERT ARKING $\ddagger$ \\ $\dagger$ Department of Biological Sciences and $\ddagger$ Institute of Gerontology, Wayne State University, Detroit, MI 48202; and \\ $\S$ Gerontological Research Center, National Institute of Aging, Baltimore, MD, U.S.A.
}

\begin{abstract}
A controlled chromosome substitution experiment was performed on a strain (NDC-L) selected for long life to determine if the genes responsible for the extended-longevity phenotype could be localized to any particular chromosome(s). All 27 different possible combinations of the three major chromosomes of Drosophila melanogaster were constructed and longevities were determined on 3875 individual animals of both sexes and analysed. The results are statistically significant and demonstrate that mean longevity is specified primarily by recessive genes on the third chromosome (c3). The extended longevity phenotype (ELP) is only expressed in those lines which are homozygous for the NDC-L type $\mathrm{c} 3$. Loci on the first (c1) and second (c2) chromosomes interact, both positively (c1) and negatively (c2), respectively, such that $\mathrm{c} 1$ represses $\mathrm{c} 2$ which in turn represses $\mathrm{c} 3$. The ELP is fully expressed in the mutual presence and mutual absence of $\mathrm{c} 1$ and $\mathrm{c} 2$. The significance of these results is discussed in the context of broader categories of molecular genetic mechanisms suggested previously to be involved in the modulation of longevity in Drosophila.
\end{abstract}

Keywords: additive inheritance, Drosophila, genetics of ageing, life span, longevity, non-additive inheritance.

\section{Introduction}

Ageing is a complex biological process. As such, the identification and characterization of the mechanisms controlling the processes of ageing have proven to be elusive. One century has passed since Weismann (1891) initiated the scientific discussion of ageing. Drosophila has been used in ageing research since 1913 (Hyde, cited in Lints \& Soliman, 1988) and yet our knowledge of the mechanisms of ageing still does not have a firm foundation.

Genetic approaches have often been used to gain insight into complex biological phenomena, primarily because of their ability to uncover a causal pathway leading from the phenotype of the gene, and vice-versa (Wilkins, 1986). There is no longer any doubt that longevity and senescence are under some form of

* Dedicated to the memory of Howard A. Schneiderman, an enthusiastic scholar, a creative administrator, and a good human being.

łCorrespondence: Robert Arking. genomic control (Johnson, 1988). However, the nature of that control remains obscure. The genetic control of ageing and longevity in Drosophila has been recently reviewed (Lints \& Soliman, 1988; Arking \& Dudas, 1989). Mutagenesis experiments for single gene life span extension mutants in Drosophila have not been successful (Roberts \& Iredale, 1985); however, experiments involving the selection of long-lived strains were successful (Rose \& Charlesworth, 1981; Rose, 1984; Luckinbill et al., 1984; Arking, 1987a). Analyses of the genetics of longevity in Drosophila indicate that longevity determinant genes with additive effects do exist; however, the effects of these genes are swamped by the large amount of non-additive genetic effects and of genotype-environment interactions (Curtsinger, 1990).

We have previously characterized and described one of these long-lived strains of Drosophila melanogaster with respect to longevity (Arking, 1987a) and various physiological (Arking et al., 1988; Pretzlaff \& Arking, 1989) and behavioural (Neslund et al., 1988) para- 
meters. This long-lived strain shows the same sequence of age-related changes as does the control strain from which it was derived, however the onset of age-related changes are significantly delayed in the former relative to the latter (Arking \& Wells, 1990). These data suggest that the ELP of this strain is a temporally altered, genetically modified normal ageing process. Such knowledge is critical to the genetic analyses of ageing processes, which are of necessity comparative analyses in which qualitative and/or quantitative comparisons are made between the gene processes taking place in long-lived strains relative to those taking place in the normal lived control strain. The two strains involved in this comparison must be carefully chosen, for if they age in different ways or if they age for different reasons, then the results of the comparisons will not be very useful and may even be misleading (Ganetzky \& Flanagan, 1978).

We describe here experiments designed to determine if the genes regulating the ELP in the long-lived NDC- $\mathrm{L}_{\mathrm{A}}(\mathrm{L})$ strain could be localized to one or more specific chromosomes by means of controlled chromosome substitution procedures and their interactions described. A preliminary report of this experiment has been published previously (Wells et al., 1987).

\section{Materials and methods}

\section{Stock construction}

The original parental lines used in this experiment are the long-lived $N D C-L_{A}(L)$ and the random bred control NDC- $\mathrm{R}_{\mathrm{A}}(\mathrm{R})$ strains of Drosophila melanogaster which have been described previously (Luckinbill et al., 1984; Luckinbill \& Clare, 1985; Arking \& Clare, 1986; Arking, 1987a,b). Randomly selected adults from these two strains were used as well as a chromosome balancer stock $\left(\operatorname{In}(2 L R) C y O, \operatorname{In}(2 L R) b w^{V I}\right.$; $\operatorname{In}(3 L R) C x D, S b$ : abbreviated herein as $C y / P m ; D / S b$ : see Lindsley \& Grell, 1967, for descriptions) obtained from Professor William Baker (then at the University of Utah), in a series of standard controlled chromosome substitution crosses (Dapkus \& Merrell, 1977) to produce a series of stable isogenic lines. These lines were generated prior to and independently of those reported by others working with this strain (Luckinbill et al., 1988). Only the first three chromosomes, which together comprise some 98 per cent of the genome of Drosophila melanogaster, are analysed in this report. The fourth chromosome was ignored for logistical and practical reasons. An example of a controlled chromosome substitution cross is shown in Fig. 1 which depicts the five-generation procedure used to generate a strain in which both of the first chromosome homo-

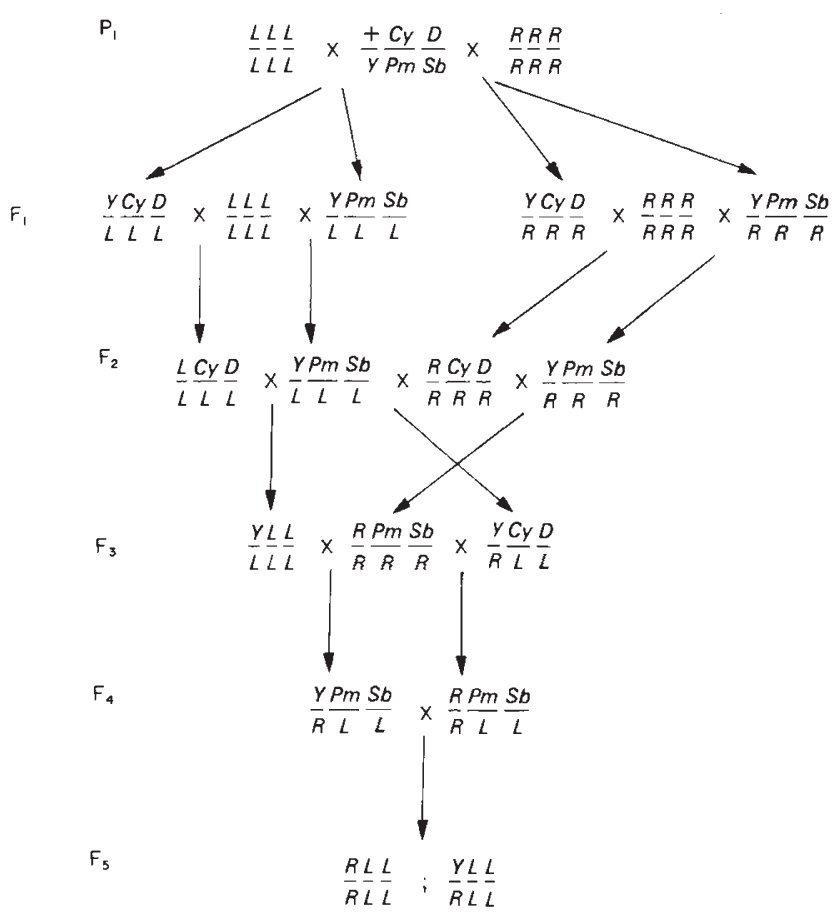

Fig. 1 A diagram of the particular crosses used to construct the 022 strain. The diagram depicts the composition of the $1 \mathrm{st}, 2 \mathrm{nd}$ and $3 \mathrm{rd}$ chromosomes, in that order from left to right, for each genotype. $L$ and $R$ symbolize chromosomes derived from the $\mathrm{L}$ and $\mathrm{R}$ strains, respectively; $Y=\mathrm{Y}$ chromosome; $\pm=$ unknown lab strain chromosome; $C y, P m$, $D$ and $S b=$ dominant markers and crossover suppressors as described in the text. The object of the crosses was to produce a specific stable combination of $\mathrm{R}$ and $\mathrm{L}$ chromosomes as shown in the $F_{5}$, without allowing them the opportunity to recombine. Analogous procedures were used to create the rest of the first six strains listed in Table 1.

logues were derived from the $\mathrm{R}$ strain while the second and third chromosome homologues were all derived from the $\mathrm{L}$ strain. This yielded a strain whose chromosome composition could be written as $R / R ; L / L ; L / L$. Similar sets of crosses (not shown) were used to generate the other stable isogenic lines, as listed in Table 1. Approximately 50 pairs were used in each of the $P_{1}$ generations, thereby avoiding homozygosity due to repeated sampling. Each of the succeeding generations usually employed $20-40$ pairs of the appropriate genotypes as parents, but never less than 10 pairs. Once established, these lines were maintained as standard laboratory populations (i.e. randomly mating populations of approximately 100 animals reproduced every few weeks) for 5-6 generations so as to facilitate stabilization before life spans were measured and before generating the remainder of the 27 lines. The notation used to identify the experimental lines throughout the remainder of this report is based on the $\mathrm{L}$ chromosome 
composition of the line. In this notation the three sequential positions in the notation represent the first, second, and third major chromosomes while the numerical value $(0,1$ or 2$)$ present at each postion represents the number of chromosomes of that set which are derived from the $\mathrm{L}$ parental strain. For example the 012 line would have the following genetic composition: $R / R ; L / R ; L / L$ females and $R / Y ; L / R ; L / L$ males. In this notation, the parental strains would be represented as $000(\mathrm{R})$ and $222(\mathrm{~L})$. Because of the $Y$ chromosome in the first position in males, the males of the $2 X X$ lines are identical to the males of the $1 X X$ lines and their results have been combined in the following tables, except as otherwise noted. The six reconstructed lines along with the two unreconstructed parental lines shown in Table 1 were then appropriately crossed so as to create the remainder of the 27 possible chromosome combinations. In all those cases where males were derived from different crosses, we took care to ensure that the $\mathrm{L}$ type first chromosomes was introduced into the zygote by a female homozygous for the L type first chromosome. This was done in order to eliminate any possible maternal effects from interfering with the analysis. We have not detected evidence of any maternal effects in other matings.

\section{Survivorship}

Survivorships were determined at $25^{\circ} \mathrm{C}$ following established procedure (Luckinbill et al., 1984; Arking, 1987a; Arking et al., 1988) using 60 pairs of animals in two replicates of 30 pairs each, one pair per vial, and changing the vials every 2 days. At these times, the viability of each member of the pair was noted and the number of eggs laid recorded. We controlled for early death due to non-ageing causes (i.e. accidents and developmental errors), by replacing any healthy and fecund animals that died within the first 2-3 weeks with the same-age siblings that were kept for that purpose. Such young animals were operationally considered to have died of a non-ageing cause if they: (i) were involved in some obvious fatal accident (i.e. crushed by the plug); or (ii) had been fecund on the day prior to their death. A decrease in fecundity has been shown to be a strong predictor of impending death (Buck et al., 1987). After this period, when one member of an experimental pair died it was replaced with a stock animal of the same sex (usually a whiteeyed animal for identification purposes) so as to keep experimental conditions the same (i.e. one pair of flies per vial) until the other member of the experimental pair died. The maintenance of constant pairs is important since it is known that life span in both sexes can vary significantly as a function of mating activity and egg production (Partridge, 1986). Individual female fecundity estimates were also made and will be described elsewhere.

The exceptions to this procedure were as follows. In one line (001) survivorships were determined on only 30 pairs of animals due to the accidental loss of one set of replicates. In the two parental lines $(000$ and 222) the vials were changed every day. This has been shown to increase life span slightly but non-significantly $(\sim 0.5$ day) when compared with the 2-day vial changes (Arking et al., 1988). Logistical reasons prevented our doing daily changes on the experimental vials. The parental measurements were done on the original (i.e. not reconstructed) 000 and 222 strains maintained under normal controlled conditions. For the 000 line $(\mathrm{R}$ parental), the life spans were measured on the $F_{37}$ $(n=60), \mathrm{F}_{43}(n=90)$ and $\mathrm{F}_{50}(n=90)$ generations. For the 222 line ( $\mathrm{L}$ parental), the life spans were measured on the $\mathrm{F}_{37}$ and $\mathrm{F}_{42}$ ( $n=60$ for both) generations. The dates of the life span assays for all lines are given below. Other departures from $n=60$ in the experimental lines were due to accidential loss of flies during vial changes but none of these included the loss of more than six animals and in most cases totalled only two or three individuals during the course of the experiment. The actual numbers of animals assayed for each genotype are listed in Table 1 and Table 3.

All life span values presented in this paper are based on the adult life span only. We have previously presented data showing that there is no correlation between the length of the developmental period and the adult life span, at least when temperature was used to double the developmental time (Arking et al., 1988). We define the latter as the span of time from eclosion to death. It is possible to generate the total life span values (i.e. the span of time from the day of egg laying to the day of adult death) needed to be able to compare meaningfully these data with those of our own earlier reports (Arking, 1987a) or with those of other investigators (Luckinbill et al., 1988) by simply adding approximately 11 days to the $\mathrm{R}$ strain adult life span values and approximately 10 days to the $L$ strain life span values presented in this report. We have not yet explored the significance of this difference in overall developmental time between the $\mathrm{R}$ and the $\mathrm{L}$ strains.

\section{Statistical analysis of temporal variation}

As it was logistically impossible to measure the life spans of all genotypes at the same time, the parental and the reconstructed lines were measured in six groups at different times beginning in November 1986 and ending in May 1988. It was therefore necessary to control for unforeseen temporal variation in life span 
across this period of time. This was accomplished by four different procedures. First, a regression analysis showed that there was no effect of assay date on mean life span in either sex (for the males, $r=-0.0687$, $t=0.379$ ( 30 d.f.), $P>0.50$; for the females, $r=0.047$, $t=0.178$ (30 d.f.), $P>0.50$ ). Secondly, we did an ANOVA on the data set as a whole which showed no significant influence of assay date on the results $(N=3845 ; F=3.35 ; P=0.067)$. Thirdly, independent empirical evidence supporting these conclusions was obtained by an independent series of controls in which we monitored the life spans of 13 generations $\left(\mathrm{F}_{34}\right.$ to $\mathrm{F}_{46}, N=23$ ) of the NDC- $\mathrm{L}_{\mathrm{A}}$ breeding stocks raised during the period of time from May 1986 to December 1988. A $t$-test showed that the mean median life span of the controls was identical to the mean of the experimental $\mathrm{L}$ lines $(t=0.084,23$ d.f., $P<0.50)$ and an $F$-test showed that the variances of the two populations were identical $\left(F_{(0.50(2), 22,1)}=20.03\right.$, $P(F \geq 20.03)=0.66)$. The two sets of data can be treated as if drawn from identical populations that are characterized by a stable life span that did not change during the course of the experiments. Finally, comparison of the several $L$ strain survival curves with the Kologomorov-Smirnov non-parametric survival test (Mode et al., 1984; Zar, 1984) showed that the several populations have survival curves which are not statistically different from one another. We concluded therefore that no unforeseen temporal variation occurred that could have biased the results of the experimental crosses.

\section{Culture conditions}

Animals were raised under high larval density conditions (i.e. $>50$ eggs per vial or equivalent) in a yeast-sucrose-agar media (supplemented with live yeast) at $25^{\circ} \mathrm{C}$ and L-D:12-12 as described previously (Luckinbill et al., 1984).

\section{Data analysis}

The data were analysed by standard statistical procedures (Zar, 1984) while procedures for certain specific tests were obtained from the literature (Dunnett 1955, 1964; Mode et al., 1984).

\section{Results}

\section{Description of female longevity}

A complete description of the female longevity data for the 27 different chromosome lines is presented in Table 1. A visual inspection of the column means suggests that the ELP appears to be associated with homozygosity for the L type third chromosome. When these data were examined using a three way fixed effect factorial analysis of variance (ANOVA) with compensation for unequal sample numbers, the results (Table 2) indicated that all three of the major chromosomes have an effect on longevity in Drosophila.

All three chromosomes, however, do not affect the ELP in the same manner. Inspection of the complete data set (Table 1) shows that chromosomes 1 and 2 have different and opposing effects on the life span through their interaction with $\mathrm{c} 3$. We describe these chromosome-specific effects by examining the data for female isogenic lines as shown in Table 1. (Analysis of the male data (Table 3 ) leads to the same conclusions.) A comparison of the mean longevities of the 222 and 002 isogenic lines shows that their longevities are not statistically different from one another $(t=1.05(178$ d.f.), $P>0.05$ ), but both are significantly different from the mean longevities of the $020(t=7.21$ (118 d.f.), $P<0.05), 200(t=4.97(178$ d.f. $), P<0.05)$ and 000 $(t=6.19$ (276 d.f.), $P<0.05)$ isogenic lines. The mean longevities of the latter two strains, however, are not statistically different from one another $(t=0.760(236$ d.f.), $P>0.05)$. The 020 strain has a very short life span which is statistically shorter than any of the other four strains (i.e. 002 vs. $020, t=7.72$ (118 d.f.), $P<0.05$ ). Thus only animals homozygous for two $L$ type third chromosomes (c3) (e.g. 002) display the ELP characteristic of the long-lived parental L (222) strain. The major genes responsible for the extended longevity phenotype must therefore be located on this chromosome (c3). Moreover, these genes are recessive (because the mean longevity of $000=$ the mean longevity of $001<$ the mean longevity of 002 ; Figs 2 and 3 ), and by themselves are both necessary and sufficient to bring about the expression of the extended longevity phenotype (because $200=220=221<002$; because $020<002$; and because $200<002$; Figs 2 and 3).

Also obvious are other lines which have two $L$ type third chromosomes but do not display the ELP. For example, a comparison of the 002 vs. the 012 or 022 life spans $(t=4.99$ ( 116 d.f. $), P<0.05)$ shows that the presence of even one $L$ type second chromosome (c2) represses the longevity enhancing effect of homozygosity for L type c3.

Moreover, further detailed inspection of the data indicates that the situation is more complex than the simple repression of $c 3$ by $c 2$. Not all strains with a $L$ type $\mathrm{c} 2$ display a shortened life span. In fact, in those lines which have both an L type c2 and a homozygous $\mathrm{L}$ type first chromosome ( $\mathrm{c} 1$ ), the life-shortening effect of $\mathrm{c} 2$ is negated (in Table 1, compare 010 vs. 210,020 vs. 220,110 vs. 210,112 vs. 212,012 vs. 212,022 vs. 222). In each case, the mean longevity of the first member of each pair is significantly different from that 
Table 1 Female longevity of each isogenic line as a function of chromosome composition*

\begin{tabular}{|c|c|c|c|}
\hline \multirow{2}{*}{$\begin{array}{l}\text { Composition of } \\
\text { chromosomes } 1 \text { and } 2 \\
\text { Females }\end{array}$} & \multicolumn{3}{|c|}{ Composition of chromosome 3} \\
\hline & --0 & --1 & --2 \\
\hline $00-$ & $\begin{array}{l}49.2 \\
47.7-50.7 \\
N=240\end{array}$ & $\begin{array}{l}47.8 \\
42.2-53.4 \\
N=30\end{array}$ & $\begin{array}{l}64.3 \\
61.3-67.3 \\
N=60\end{array}$ \\
\hline $01-$ & $\begin{array}{l}40.8 \\
37.9-43.7 \\
N=60\end{array}$ & $\begin{array}{l}42.3 \\
39.1-45.6 \\
N=60\end{array}$ & $\begin{array}{l}52.5 \\
48.6-56.4 \\
N=58\end{array}$ \\
\hline $02-$ & $\begin{array}{l}44.8 \\
41.0-48.6 \\
N=60\end{array}$ & $\begin{array}{l}50.3 \\
46.7-53.9 \\
N=60\end{array}$ & $\begin{array}{l}49.9 \\
46.6-53.1 \\
N=60\end{array}$ \\
\hline $10-$ & $\begin{array}{l}46.2 \\
43.3-49.0 \\
N=60\end{array}$ & $\begin{array}{l}53.3 \\
49.9-56.7 \\
N=60\end{array}$ & $\begin{array}{l}59.3 \\
55.8-62.8 \\
N=60\end{array}$ \\
\hline $11-$ & $\begin{array}{l}43.4 \\
40.3-46.5 \\
N=60\end{array}$ & $\begin{array}{l}52.9 \\
50.7-55.2 \\
N=180\end{array}$ & $\begin{array}{l}51.5 \\
47.4-55.5 \\
N=58\end{array}$ \\
\hline $12-$ & $\begin{array}{l}50.5 \\
47.0-54.0 \\
N=60\end{array}$ & $\begin{array}{l}52.3 \\
47.3-55.2 \\
N=60\end{array}$ & $\begin{array}{l}54.4 \\
50.5-58.4 \\
N=60\end{array}$ \\
\hline $20-$ & $\begin{array}{l}51.7 \\
48.7-54.6 \\
N=60\end{array}$ & $\begin{array}{l}52.8 \\
49.1-56.5 \\
N=59\end{array}$ & $\begin{array}{l}63.4 \\
60.9-65.9 \\
N=60\end{array}$ \\
\hline $21-$ & $\begin{array}{l}48.1 \\
44.3-52.0 \\
N=60\end{array}$ & $\begin{array}{l}52.2 \\
48.5-55.9 \\
N=60\end{array}$ & $\begin{array}{l}62.7 \\
58.4-66.9 \\
N=60\end{array}$ \\
\hline $22-$ & $\begin{array}{l}54.6 \\
51.3-58.0 \\
N=60\end{array}$ & $\begin{array}{l}56.1 \\
51.8-60.5 \\
N=60\end{array}$ & $\begin{array}{l}62.1 \\
59.5-64.6 \\
N=120\end{array}$ \\
\hline Column means & $\begin{array}{l}48.1 \\
47.8-48.3\end{array}$ & $\begin{array}{l}51.6 \\
51.3-51.9\end{array}$ & $\begin{array}{l}58.3 \\
57.8-58.8\end{array}$ \\
\hline
\end{tabular}

*Data is shown as the mean, the $95 \%$ confidence limits, and the number of animals assigned.

of the second member of the pair. There are no observed exceptions to this trend.

The effects of dosage compensation on $\mathrm{c} 1$ is shown by the fact that 122 males have an $\mathrm{L}$ type life span but that 122 females have a $\mathrm{R}$ type life span, which in turn is statistically identical to that of the 022 female (i.e. $122 \mathrm{f}=022 \mathrm{f}<222 \mathrm{f}=122 \mathrm{~m}$; Figs 2 and 3 ).

Thus the net conclusion of these data is that $\mathrm{c} 1$ represses $\mathrm{c} 2$ which in turn represses the expression of the longevity enhancing genes on $\mathrm{c} 3$.

\section{Description of male longevity}

The complete description of the male longevity data for the 18 different chromosome lines is shown in Table 3. An ANOva was performed on these data and the results (Table 4) also indicate that all three of the major chromosomes have an effect on the longevity of Drosophila. However, the same cautions mentioned above apply here as well, for it is clear that only chromosome combinations in which the animal is homozygous for the L type c 3 display the ELP (compare $002,102,112,112$ ) while combinations involving the L type $\mathrm{c} 2$ and $\mathrm{c} 3$ chromosomes do not display this phenotype (compare 012,022). It is also clear that animals possessing an $\mathrm{L}$ type $\mathrm{c} 1$ and $\mathrm{L}$ type $\mathrm{c} 2$ do not show the decreased life span characteristic of those animals possessing only the L type $\mathrm{c} 2$ (compare 111 vs. $011 ; 122$ vs. $022 ; 122$ or 112 vs. 012). Thus the male longevity data also lead to the conclusions that $(\mathrm{a})$, the genes on $\mathrm{c} 3$ are necessary and essential for the expression of the extended longevity phenotype; and (b), the 
Table 2 ANOVA of the female longevity data*

\begin{tabular}{|c|c|c|c|c|c|}
\hline Source of variation & SS & d.f. & MS & $F$ & $P$ \\
\hline Within cells & $379,575.66$ & 2039 & 186.16 & & \\
\hline Constant & $46,792,925.69$ & 1 & $46,792,925.7$ & $25,101.97$ & 0.000 \\
\hline Chromosome 1 & $13,273.77$ & 2 & $6,636.88$ & 35.65 & 0.000 \\
\hline Chromosome 2 & $4,719.01$ & 2 & $2,359.50$ & 12.67 & 0.000 \\
\hline Chromosome 3 & $29,439.50$ & 2 & $14,719.75$ & 79.07 & 0.000 \\
\hline $\begin{array}{l}\text { Chromosome } 1 \text { by } \\
\text { Chromosome } 2\end{array}$ & $3,673.99$ & 4 & 918.50 & 4.93 & 0.001 \\
\hline $\begin{array}{c}\text { Chromosome } 1 \text { by } \\
\text { Chromosome } 3\end{array}$ & $5,169.51$ & 4 & $1,292.38$ & 6.94 & 0.000 \\
\hline $\begin{array}{c}\text { Chromosome } 2 \text { by } \\
\text { Chromosome } 3\end{array}$ & $4,506.52$ & 4 & $1,126.63$ & 6.05 & 0.000 \\
\hline $\begin{array}{l}\text { Chromosome } 1 \text { by } \\
\text { Chromosome } 2 \text { by } \\
\text { Chromosome } 3\end{array}$ & $4,267.80$ & 8 & 533.48 & 2.87 & 0.004 \\
\hline
\end{tabular}

*Three-way fixed effects factoral ANOVA with compensation for unequal sample number.

Table 3 Male longevity as a function of chromosome composition*

\section{Composition of}

chromosomes 1 and 2

\begin{tabular}{llll}
\hline Males & --0 & --1 & --2 \\
\cline { 2 - 4 } $00-$ & 52.3 & 44.7 & 67.7 \\
& $50.9-53.7$ & $38.6-50.8$ & $65.1-70.2$ \\
$01-$ & $N=240$ & $N=27$ & $N=60$ \\
& 48.6 & 48.6 & 52.3 \\
$02-$ & $46.0-51.1$ & $45.7-51.5$ & $48.5-56.1$ \\
& $N=60$ & $N=60$ & $N=54$ \\
$10-$ & 54.9 & 54.6 & 59.4 \\
& $51.2-58.6$ & $51.8-57.5$ & $56.3-62.4$ \\
$11-$ & $N=60$ & $N=60$ & $N=60$ \\
& 49.4 & 47.5 & 63.5 \\
$12-$ & $47.1-51.8$ & $44.8-50.2$ & $61.7-65.3$ \\
& $N=117$ & $N=116$ & $N=120$ \\
& 52.1 & 57.4 & 64.4 \\
Column means & $50.2-54.0$ & $55.6-59.3$ & $60.4-67.4$ \\
& $N=120$ & $N=237$ & $N=119$ \\
& 57.8 & 57.2 & 66.2 \\
& $55.7-59.9$ & $54.7-59.6$ & $64.1-68.3$ \\
& $N=120$ & $N=120$ & $N=180$ \\
& 52.6 & 53.8 & 63.5 \\
& $52.3-52.9$ & $53.6-54.0$ & $63.1-63.9$ \\
\hline
\end{tabular}

*Data is shown as the mean, the $95 \%$ confidence limits, and the number of animals assigned.

expression of $\mathrm{c} 3$ is repressed by $\mathrm{c} 2$ which in turn is repressed by $\mathrm{c} 1$. Males and females show the same patterns of chromosome interactions.

As mentioned in the 'Materials and methods', some of the male chromosome lines have mean values which represent the pooled longevities of individuals derived from different types of crosses (i.e. 112 males were derived from both a 202 female $\times 022$ male cross and from a 202 female $\times 222$ male cross). Our records allowed us to identify and analyse separately the nine 
Fig. 2 The chromosome constitution and the mean life span are shown for females of each of the isogenic lines listed in Table 1. The survival curves and the mean life span of each line were compared with those of the 000 and 222 lines, and the statistical results were used to group the lines as described in the text. In most cases, the parametric and non-parametric tests yielded the same conclusions for each line; the astericks indicate those cases where the two tests gave different results (see text for explanation). Note that the nonparametric results yield the more stringent grouping, and that is what is shown here.

Fig. 3 The chromosome constitution and the mean life span are shown for males of each of the isogenic lines listed in Table 3. The survival curves and the mean life span of each line were compared with those of the 000 and 222 lines, and the statistical results were used to group the lines.
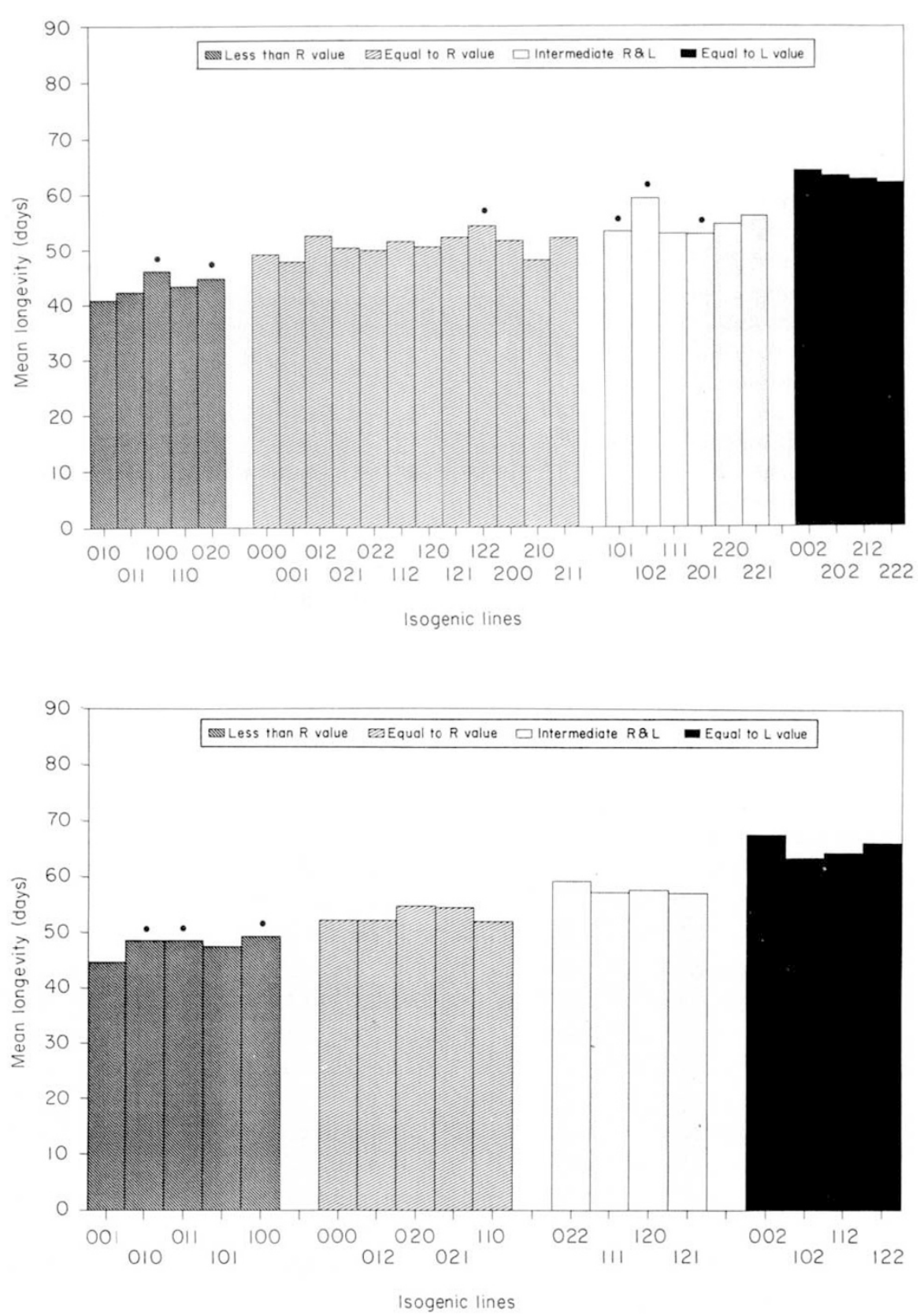

groups of affected males. Only one of these nine sets (the 100 males) was found to be composed of populations derived from different crosses which were significantly different from one another. We therefore doubt that the difference in lineage can fully explain the intermediate male values noted in Table 3 .

\section{Statistical analysis of longevity}

Both sexes show the same sort of longevity response to the same sort of chromosome combinations. There is no evidence of heterosis. Both non-parametric and parametric tests were used to analyse statistically each of the 27 individual genotypes to determine the specific effects of chromosome composition on longevity. We employed the Kologmorov-Smirnov non-parametric survival test (Mode et al., 1984; Zar, 1984) to compare individually the survival curves of each sex of each isogenic line with the same-sex survival curve of the 000 and the 222 strain. This procedure allowed us to determine the statistical similarity of each line relative to both controls. Most, but not all, of the lines are statistically similar to one of the control lines and statistically dissimilar from the other control line. There are, however, several important exceptions to this general rule. For ease of comparison, these results 
Table 4 AnOva of the male longevity data*

\begin{tabular}{|c|c|c|c|c|c|}
\hline Source of variation & SS & d.f. & MS & $F$ & $P$ \\
\hline Within cells & $316,772.19$ & 1909 & 165.94 & & \\
\hline Constant & $4,349,644.67$ & 1 & $4,349,644.7$ & $26,212.75$ & 0.000 \\
\hline Chromosome 1 & $4,600.82$ & 1 & $4,600.82$ & 27.73 & 0.000 \\
\hline Chromosome 2 & $6,011.98$ & 2 & $3,005.99$ & 18.12 & 0.000 \\
\hline Chromosome 3 & $32,645.25$ & 2 & $16,322.63$ & 98.37 & 0.000 \\
\hline $\begin{array}{l}\text { Chromosome } 1 \text { by } \\
\text { Chromosome } 2\end{array}$ & $5,334.09$ & 2 & $2,667.05$ & 16.07 & 0.000 \\
\hline $\begin{array}{c}\text { Chromosome } 1 \text { by } \\
\text { Chromosome } 3\end{array}$ & $1,152.00$ & 2 & 576.00 & 3.47 & 0.031 \\
\hline $\begin{array}{c}\text { Chromosome } 2 \text { by } \\
\text { Chromosome } 3\end{array}$ & $8,444.22$ & 4 & $2,111.05$ & 12.72 & 0.000 \\
\hline $\begin{array}{l}\text { Chromosome } 1 \text { by } \\
\text { Chromosome } 2 \text { by } \\
\text { Chromosome } 3\end{array}$ & $1,772.51$ & 4 & 443.13 & 2.67 & 0.004 \\
\hline
\end{tabular}

*Three-way fixed effects factoral ANOVA with compensation for unequal sample number.

are shown in Fig. 2 where they have been arranged according to whether the survival curve for each particular chromosome line had a longevity which was statistically greater than, less than, identical to or intermediate between that of the $000(\mathrm{R})$ and the $222(\mathrm{~L})$ controls. It may be seen that the female data fall into one of four statistically discrete classes; a shorter lived type, an $\mathrm{R}$ type, an intermediate type or an $\mathrm{L}$ type longevity. The production of each particular life span phenotype is clearly governed by the genotypes of their chromosome combinations. The data for males (Fig. 3) fall into the same general classification scheme.

The grouping into four mean life span categories based on the overall survival data was verified by using the Dunnett's multiple comparison parametric test in which multiple sample means are individually compared with a control mean and their statistical similarity/dissimilarity ascertained (Dunnett, 1955, 1964; Zar, 1984). The mean longevity value for each sex of each chromosome line (Tables 1 and 3) was independently compared with the mean longevity of the 000 and the 222 control lines. The data were analysed using the same logic as for the non-parametric tests described above. The two sets of conclusions are mostly compatible, the non-parametric test being the more stringent. The tests disagree in only eight of the 82 comparisons, and these cases are indicated by an asterisk in Figs 2 and 3. In all but two cases (101 and 201), the disagreements involved a drop to the next lowest life span category. In no case did either test statistically associate an extended life span with animals which were not homozygous for the $\mathrm{L}$ type third chromosome.

\section{Discussion}

All of the 27 possible chromosome combinations were examined in an effort to determine if the longevity determinant genes believed responsible for the ELP in our NDC-L strain could be localized to any particular chromosome or set of chromosomes. The results are quite clear. First, longevity is a quantitative trait in which the ELP is only expressed in those lines which are homozygous for the L type c3. In addition, there exist loci on $\mathrm{c} 2$ which have a negative regulatory effect on the $c 3$ genes. This inhibitory effect of $c 2$ is itself inhibited by homozygous or hemizygous $\mathrm{L}$ type $\mathrm{c}$. Taken together, these chromosomal interactions defined which genotypes can, or cannot, express the extended longevity phenotype when raised under the appropriate environmental conditions (see companion paper).

\section{Genetic components affecting longevity}

Curtsinger (1990) has examined the nature of the genetic components affecting life span in large inbred and hybrid laboratory populations of Drosophila. He has estimated that about 20 per cent of the total genetic variance associated with life span in these populations is due to additive genetic effects, about 20 per cent of the variance is due to non-additive genetic or interaction effects, and about 60 per cent of the total variance is due to genotype-environmental interactions. Our analysis has allowed us to identify these same components, albeit in a different manner. We have clearly identified a set of recessive genes on $c 3$ 
whose presence is required for the expression of the extended longevity phenotype and which presumably corresponds to the additive genetic effects. We have also described above positive and negative interaction effects of c1 and c2 upon c3; these epistatic chromosomal interactions presumably correspond to the nonadditive genetic variance. Finally, the companion paper (Buck et al., 1993) deals with the genotype-environment interactions involved in the expression of the extended longevity phenotype. We have found no evidence for heterosis.

This classification of the different types of genetic effects observed in the system suggests that any further understanding of the genetic components affecting longevity in the $\mathrm{L}$ strain must involve an analysis of these three classes of effects.

Longevity determinant genes. Once the non-additive and environment effects are held constant, then it is apparent that the animals' life span is under the control of the recessive genes on $\mathrm{c} 3$. If these $\mathrm{c} 3$ genes in this strain are analogous to the longevity determinant genes described by Cutler (1975) and by Sacher (1975), then there is probably more than one set of longevity determinant genes. Two of the existing three extended longevity strains known to exist in Drosophila were derived by similar experimental protocols in which the founder stock was directly selected for delayed reproduction and only indirectly selected for long life (Rose, 1984; Luckinbill et al., 1984). Other successful selection schemes, particularly if they affected other phases of the adult life cycle, might well depend on longevity determinant genes other than those characterized in this report (Hoffmann \& Parsons, 1992; Rose et al., 1992). A thorough understanding of the genetic regulation of the processes of ageing will depend on our knowing just how many different components can comprise the genetic system governing longevity.

Our data should not be interpreted as implying that the only genes involved in the expression of the extended longevity phenotype are these $c 3$ genes. The present data can be interpreted as suggesting that the action of the $\underline{\mathrm{c} 3}$ genes precedes, and is epistatic to, the action of genes elsewhere in the genome. In the absence of the $\mathrm{L}$ type $\mathrm{c} 3$ genes, these other genes demonstrably are not effective in producing the ELP (compare, for example, 220 vs. 002 in Fig. 2). It appears that there is a temporal hierarchy of molecular genetic events which are expressed beginning in the third larval instar (Dudas \& Arking, in preparation; also see companion paper of Buck et al., 1993) and which culminate in the delayed onset of senescence in the adult phases of the life span (Arking \& Wells, 1990).
In an independent set of experiments, we have examined the electrophoretic patterns of several enzymes in the $\mathrm{R}$ and $\mathrm{L}$ strains (Dudas et al., in preparation). The alcohol dehydrogenase $(A D H)$ gene is located at 50.1 on $\mathrm{c} 3$ and plays a role in the expression of the ELP (Dudas \& Arking, in preparation). The pertinent observation in this system is that the electrophoretic pattern of the ADH isozymes is dependent only on the $\mathrm{L}$ or $\mathrm{R}$ origin of the $\mathrm{c} 3$, the origin of the $\mathrm{c} 2$ being of no consequence. This result confirms the existence in our system of trans-acting chromosomal interactions.

Chromosomal interactions. The chromosomal interactions described above suggest that the simplest form of the genetic circuits involved in modulating the effect of the longevity determinant genes are: (i) the $\mathrm{c} 2$ inhibits the $\mathrm{c} 3$ genes essential for the expression of the ELP; and (ii), the c2 is itself inhibited by either homozygous or hemizygous $\mathrm{c} 1$. Thus, the net effect of an animal being homozygous for $\mathrm{c} 1$ and $\mathrm{c} 2$ is to allow $\mathrm{c} 3$ to be expressed (222). The $\mathrm{c} 3$ is also expressed if the animal contains only $\mathrm{R}$ type $\mathrm{c} 1$ and $\mathrm{c} 2(002)$. Consequently, balanced combinations (i.e. 202, 212) of $\mathrm{c} 1$ and c2 yield an L type life span; unbalanced combinations of $\mathrm{c} 1$ and $\mathrm{c} 2$ yield an $\mathrm{R}$ type life span (Figs 2 and $3)$. The fitness effects of these properties of the L type c1 and c2 have not been investigated. We do not know why the selection procedure did not directly select just for the $\mathrm{c} 3$ containing the longevity enhancing genes. We know that our longevity selection regime was inadvertently structured so as to simultaneously select for fast larval development under NDC conditions. It is possible that this parameter may have something to do with the presence of the chromosomal interactions.

Environmental effects. Environmental factors can have a specific or non-specific effect on the ageing processes. Temperature is an example of a non-specific environmental factor. It has long been known that lowering the ambient temperature will prolong the life span of poikilothermic animals. We have shown (Arking et al., 1988) that both the $\mathrm{L}$ and the $\mathrm{R}$ strains react as expected, both showing an inverse relationship between temperature and longevity. However, at any temperature, the $\mathrm{L}$ strain always has a higher mean and maximum life span relative to the $\mathrm{R}$ strain. Temperature non-specifically affects longevity without specifically affecting the molecular genetic processes responsible for the regulation of ageing.

Clare \& Luckinbill (1985) have reported that larval density appeared to specifically affect the longevity of the $\mathrm{L}$ strain. We have examined this in some detail and conclude that larval density is indeed a specific modu- 
lator of ageing in Drosophila. This work is described in detail in the companion paper of Buck et al. (1993).

\section{Comparison with previous studies}

An Oregon- $R$ population of Drosophila has been reported to show large and presumably non-random variations in their mean life span values when they are continuously assayed over long periods of time (Lints \& Hoste, 1979; Lints et al., 1989). The experiments described here took approximately 2 years to complete; they could clearly have been subjected to these same undefined and inexplicable variations. However, this is not likely to be the case as the statistical control data, presented in the 'Materials and methods' section above, shows no temporal variation in the values.

Rose and his colleagues have also selected five longlived strains of Drosophila (Rose \& Charlesworth, 1981; Rose, 1984), and have used various types of population crosses to perform a quantitative genetic analysis of them (Hutchinson \& Rose, 1991; Hutchinson et al., 1991). These latter studies enabled them to conclude that: (i) the extended longevity phenotypes involve additive genetic inheritance in population crosses (although they cannot exclude the presence of dominance among individual loci); (ii) c3 is involved (Rose, cited in Finch, 1990, pp. 307-308); (iii) there are a number of other physiological traits that are associated with extended longevity; and (iv) there is very little difference between each of the several control or long-lived lines. The data presented in this report and the data presented in Arking \& Wells (1990) are consistent with each of their first three conclusions. Their conclusions regarding the genetic similarity between independent replicate lines is important and reassuring, especially since logistical considerations have kept us from analysing independent replicate lines, and suggests that these results may be of some general applicability. The data and interpretations from their experiments and from our experiments are consistent with each other.

Yonemura et al. (1989) used two inbred lines of $D$. melanogaster, each characterized by very different mean longevities, to investigate the genetic basis for longevity in those strains. They have concluded, by analysis of $F_{1}, F_{2}$, and backcross life spans, that the observed pattern of longevities could best be explained by the existence of allelic differences between the two strains at an unspecified autosomal locus and at a sexlinked locus. Their data are consistent with ours inasmuch as we have demonstrated recessive genes on $\mathrm{c} 3$ that are necessary and sufficient for the expression of the ELP, as well as the repressive effect of $\mathrm{c} 1$ on $\mathrm{c} 2$ that accounts for the statistical association of $\mathrm{c} 1$ with the
ELP. Their report of autosomal overdominance is not confirmed by our results. It is possible, however, that their statistical approach to the problem has led them to interpret the segregation of the $L$ type $c 1, c 2$, and $c 3$ into the four balanced genotypes (Fig. 2) as being overdominant relative to the six unbalanced intermediate genotypes (Fig. 2). If so, then the difference in our results is simply the outcome of the different statistical and genetic methods employed.

A genetic analysis of the same NDC-L strain reported here has been previously reported by Luckinbill et al. (1987, 1988). Their initial studies suggested that the ELP was due to the effects of one Iocus (Luckinbill et al., 1987). In a subsequent paper (Luckinbill et al., 1988), they reported that longevity varied continuously across a range of values defined by the two parental strains and concluded that it was under polygenic control with contributing elements on all three major chromosomes. Most of the effect (i.e. about 72 per cent) resided on the third chromosome and about 17 per cent was localized to the first chromosome. This data is consistent with our ANOvA results. Our data presented here extend these results by demonstrating that the ELP is dependent not only upon the composition of $\mathrm{c} 3$ but that both positive and negative chromosomal interactions are also involved.

The overall agreement among these several studies is noteworthy. Four laboratories used different techniques to analyse three different long-lived strains of Drosophila melanogaster and all have demonstrated that the main genetic effect responsible for the ELP is autosomal and primarily involves $\mathrm{c} 3$.

\section{Conclusion}

Martin \& Turker (1988) have pointed out that a genetic analysis of ageing is the approach most likely to uncover the causal mechanisms underlying normal biological ageing. The questions that must be initially answered in such an approach are: (i) the determination of the location, number and type of genes involved; and (ii) the nature of the gene action implicated in such systems. In this report, we have used chromosomal substitution to demonstrate that, in both sexes of our Drosophila strains, the ELP is expressed only in four genotypes $(002,202,212$, and 222; see Fig. 2) which are homozygous for the L type $\mathrm{c} 3$. Loci on $\mathrm{c} 1$ and $\mathrm{c} 2$ interact, both positively (c1) and negatively (c2) such that $\mathrm{c} 1$ represses $\mathrm{c} 2$ which in turn represses $\mathrm{c} 3$. Trans-acting interchromosomal effects play an important part in the genetic control of the ELP. Our companion paper (Buck et al., 1993) extends these findings so as to define the time of action of these genes as well as to suggest at least two potential physiological mechanisms 
which may be involved in the expression of the ELP. The 23 other genotypes exhibit a spectrum of 'normal' longevity values which do not demonstrate any statistical significant extension of longevity. These 23 genotypes appear to represent normal plasticity of the baseline $\mathrm{R}$ strain to genetic alterations.

A careful genetic analysis at the classical level has been demonstrated in other developmental systems to be a prerequisite for a successful analysis at the molecular level. This type of genetic analysis will also lay the basis for potential intervention strategies. The information presented in this report provides part of the baseline for future work on specific molecular genetic mechanisms of ageing processes.

\section{Acknowledgements}

We thank Mr Chris Kolde and Ms Rehka Patel for their efforts and assistance during the construction of the six isogenic lines. We would also like to thank Ms Susan Lagrou, Ms Vesna Evkoska and Mr Leonard Karadimas for their assistance during the life span measurements, and Ms Christine Neslund for her general technical assistance. The comments of T. E. Johnson on an earlier version of this manuscript were very helpful and are appreciated, as are the comments of Michael Nicholson and Allan Force. This research was supported by the WSU Institute of Gerontology, by a WSU-NIH Biomedical Research Support Award to R.A., and by a WSU President's Excellence Award to R.A.

\section{References}

ARKING, R. 1987a. Successful selection for increased longevity in Drosophila: analysis of the survival data and presentation of a hypothesis on the genetic regulation of longevity. Exp. Gerontol., 22, 199-220.

ARKING, R. 1987b. Letter to the editor. Exp. Gerontol., 22, 223-226.

ARKING, R., BUCK, S., WELLS, R. A. AND PRETZLAFF, R. 1988. Metabolic rates in genetically based long lived strains of Drosophila. Exp. Gerontol., 23, 59-76.

ARKING, R. AND DUDAS, S. P. 1989. A review of genetic investigations into ageing processes in Drosophila. J. Amer. Geriatrics Soc., 37, 757-773.

ARKING, R. AND WELLS, R. A. 1990. Genetic alteration of normal ageing processes is responsible for extended longevity in Drosophila. Devel. Genetics, 11, 141-148.

BAKER, G. T., III AND SPROTT, R. L. 1988. Biomarkers of ageing. Exp. Gerontol., 23, 223-239.

BUCK, S., WELLS R. A. AND ARKING, R. 1987. A biomarker of senescence and impending death in Drosophila. The Gerontologist, 27, 149A (abstract).
BUCK, S., NICHOLSON, M., DUDAS, S. P., BAKER, G. T., III AND ARKING, R. 1993. Larval regulation of adult longevity in a genetically selected long lived strain of Drosophila melanogaster. Heredity, 71, 23-32.

ClARE, M. AND LuCKINBILL, L. 1985. The effects of geneenvironment interaction on the expression of longevity. Heredity, 55, 19-29.

CURTSINGER, J. 1990. Genetic and environmental components in variance of longevity in Drosophila males. Talk presented at 43rd annual meeting of Gerontology Soc. of America, 18 November.

CUTLER, R. G. 1975. Evolution of human longevity and the genetic complexity governing ageing rate. Proc. Natl. Acad. Sci. U.S.A., 72, 4664-4668.

DAPKUS, D. AND MERRELL, D. J. 1977. Chromosomal analysis of DDT resistance in a long term selected population of Drosophila melanogaster. Genetics, 87, 685-697.

DUDAS, S. P. AND ARKING, R. 1990. Genetic expression in long lived strains of Drosophila. The Gerontologist, 30, 115A (abstract).

DUNNETT, C. w. 1955. A multiple comparison procedure for comparing several treatments with a control. Am. Stat. Assoc. J., (Dec.), 1096-1121.

DUNNETT, C. W. 1964. New tables for multiple comparisons with a control. Biometrics, (Sept.), 482-491.

FINCH, C. 1990. Longevity, Senescence, and the Genome. University of Chicago Press, Chicago, IL.

GANETZKY, B. AND FLANAGAN, J. R. 1978. On the relationship between senescence and age-regulated changes in two wild-type strains of Drosophila melanogaster. Exp. Gerontol., 13, 189-196.

HOFFMANN, A. AND PARSONS, P. 1992. Selection for adult dessication resistance in Drosophila melanogaster: Fitness components, larval resistance and stress correlations. Biol. J. Linn. Soc., in press.

HUTCHINSON, E. W. AND ROSE, M. R. 1991. Quantitative genetics of postponed ageing in Drosophila melanogaster. I. Analysis of outbred populations. Genetics, 127, 719-727.

HUTCHINSON, E. W., SHAW, A. J. AND ROSE, M. R. 1991. Quantitative genetics of postponed ageing in Drosophila melanogaster. II. Analysis of selected lines. Genetics, 127, 729-737.

JOHNSON, T. E. 1988. Genetic specification of lifespan: processes, problems and potentials. J. Gerantol, Biol. Sci., 43, B 87-92.

LINDSLEY, D. L. AND GRELL. E. H. 1967. Genetic variations of Drosophila melanogaster. Carnegie Inst. of Washington Publ. No. 627.

LINTS, F. A. AND HOSTE, C. 1979. The Lansing effect revisited I. Life span. Exp. Gerontol, 9, 51-69.

LiNTS, F. A. AND Soliman, H. M. (eds.) 1988. Drosophila as a Model Organism for Ageing Studies. Blackie and Son Ltd., Glasgow.

LINTS, F. A., LINTS, C. V., BULLENS, R., BOURGERS, M. AND DELINCE, J. 1989. Unexplained variations in life span of the Oregon- $R$ strain of Drosophila melanogaster over a four-year period. Exp. Gerontol., 24, 265-271.

LUCKINBILL, L. S., ARKING, R., CLARE, M. J., CIROCCO, W. C. AND BUCK, s. A. 1984. Selection for delayed senescence in Drosophila melanogaster. Evolution, 38, 996-1004. 
LUCK1NBILL, L. S. AND CLARE, M. J. 1985. Selection for life span in Drosophila melanogaster. Heredity, 55, 9-18.

LUCKINBILL, L. S., CLARE, M. J., KRELL, W. L., CIROCCO, W. C. AND RICHARDS, P. 1987. Estimating the number of genetic elements that defer senescence in Drosophila. Evol. Ecol., 1, $37-46$.

LUCKINBILL, L. S., GRAVES, J. L., REED, A. H. AND KOETSAWANG, S. 1988. Localizing genes that defer senescence in Drosophila melanogaster. Heredity, 60, 367-374.

MARTIN, G. M. AND TURKER, M. S. 1988. Model systems for the genetic analysis of mechanisms of ageing. J. Gerontol. Biological Sciences, 43, B33-39.

MODE, C. J., ASHLEIGH, R. D., ZAWODNIAK, A. AND BAKER, G. T., III. 1984. On statistical tests of significance in studies of survivorship in laboratory animals. J. Gerontology, 39, $36-42$

NESLUND, C. M., WELlS, R. A. AND ARKING, R. 1988. Behavioural genetic analysis of a long lived strain of Drosophila melanogaster. The Gerontologist, 28, 226A.

PARTRIDGE, L. 1986. Sexual activity and life span. In: K. G. Collatz and R. S. Sohal (eds) Insect Ageing: Strategies and Mechanisms, Springer-Verlag, Berlin, pp. 46-54.

PRETZlaf, R. AND ARKING, R. 1989. Patterns of amino acid incorporation in long lived genetic strains of Drosophila melanogaster. Exp. Gerontol, 24, 67-81.

ROBERTS, P. A. AND IREDALE, R. B. 1985. Can mutagenesis reveal major genes affecting senescence? Exp. Gerontol., 20, $119-121$.
ROSE, M. R. 1984. Laboratory evolution of postponed senescence in Drosophila melanogaster. Evolution, 38, 1004-1010.

ROSE, M. R. AND CHARLESWORTH, B. 1981. Genetics of life history in Drosophila melanogaster. II. Exploratory selection experiments. Genetics, 97, 187-196.

ROSE, M. R., VU, L. N., PARK. S. U. AND GRAVES, JR., J. L. 1992. Selection on stress resistance increases longevity in Drosophila melanogaster. Exp. Gerontol., 27, 241-250.

SACHER, G. A. 1975. Maturation and longevity in relation to cranial capacity in hominid evolution. In: Tuttle, R. (ed.) Antecedents of Man and After: Vol. 1, Primates: Functional Morphology and Evolution. Mouton, The Hague, pp. 419-441.

WELLS, R., BUCK, A. S., ALI, R., MARZOUQ, O. AND ARKING, R. 1987. Localization of the longevity genes in $D$. melanogaster. The Gerontologist, 27, 149A (abstr.)

weismann, A. 1891. Essays on Heredity. Calvender Press, Oxford.

wiLkins, A. S. 1986. Genetic Analysis of Animal Development. John Wiley and Sons, New York.

YONEMURA, I., MOTOYAMA, T. AND HASEKURA, H. 1989. Mode of inheritance of major genes controlling the life span differences between two inbred strains of Drosophila melanogaster. Hereditas, 111, 207-214.

ZAR, J. H. 1984. Biostatistical Analysis, 2nd edn. Prentice-Hall, Englewood Cliffs, New Jersey. 